\title{
Erratum to: Inhibitors of Succinate: Quinone Reductase/ Complex II Regulate Production of Mitochondrial Reactive Oxygen Species and Protect Normal Cells from Ischemic Damage but Induce Specific Cancer Cell Death
}

Stephen J. Ralph • Rafael Moreno-Sánchez • Jiri Neuzil • Sara Rodríguez-Enríquez

Published online: 13 September 2011

(C) Springer Science+Business Media, LLG 2011

\section{Erratum to: Pharm Res \\ DOI 10.1007/s11095-011-0566-7}

The abstract to this article should have read as follows:

Succinate:quinone reductase (SQR) of Complex II occupies a unique central point in the mitochondrial respiratory system as a major source of electrons driving reactive oxygen species (ROS) production. It is an ideal pharmaceutical target for modulating ROS levels in normal cells to prevent oxidative stress-induced damage or alternatively, increase ROS in cancer cells, inducing cell death. The value of drugs like diazoxide to prevent ROS production, protecting normal cells, whereas vitamin $\mathrm{E}$ analogues promote ROS in cancer cells to kill them is highlighted. As pharmaceuticals these agents may prevent degenerative disease and their modes of action are presently being fully explored. The evidence that SDH/Complex II is tightly coupled to the NADH/NAD+ ratio in all cells, impacted by the available supplies of Krebs cycle intermediates as essential NAD-linked substrates, and the NAD+-dependent regulation of SDH/Complex II are reviewed, as are links to the $\mathrm{NAD+-dependent}$ dehydrogenases, Complex I and the E3 dihiydrolipoamide dehydrogenase to produce ROS. This review collates and discusses diverse sources of information relating to ROS production in different biological systems, focussing on evidence for SQR as the main source of ROS production in mitochondria, particularly its relevance to protection from oxidative stress and to the mitochondrial-targeted anticancer drugs (mitocans) as novel cancer therapies.

The online version of the original article can be found at http://dx.doi.org/ | 0. 1007/s | |095-0 | |-0566-7.

\footnotetext{
S. J. Ralph $(\bowtie) \cdot J$. Neuzil

School of Medical Science \& Health Research Institute

Griffith University

Gold Coast, QLD, Australia 4222

e-mail: s.ralph@griffith.edu.au

R. Moreno-Sánchez ·S. Rodríguez-Enríquez

Departamento de Bioquímica

Instituto Nacional de Cardiología

Tlalpan México, DF 14080, Mexico

J. Neuzil

Molecular Therapy Group, Institute of Biotechnology

Academy of Science of the Czech Republic

Prague 4 I42 20, Czech Republic
} 